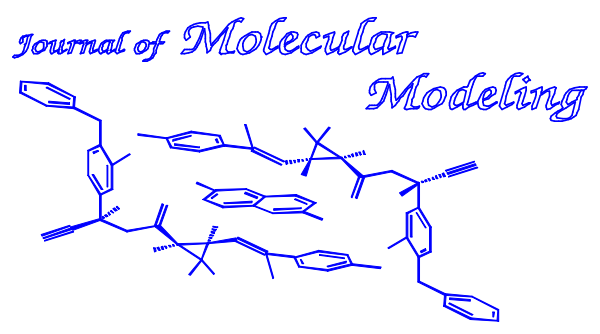

(C) Springer-Verlag 1996

\title{
A Semiempirical Transition State Structure for the First Step in the Alkaline Hydrolysis of Cocaine. Comparison between the Transition State Structure, the Phosphonate Monoester Transition State Analog, and a Newly Designed Thiophosphonate Transition State Analog
}

\author{
Edward C. Sherer, Gordon M. Turner, Tricia N. Lively, Donald W. Landry",* and George C. Shields* \\ Department of Chemistry, Lake Forest College, Lake Forest, Illinois 60045 USA (george@sgbq.bq.ub.es) \\ $\dagger$ Department of Medicine, Columbia University College of Physicians and Surgeons, New York, NY 10032 USA
}

Received: 30 January 1996 / Accepted: 29 March 1996 / Published: 19 April 1996

\begin{abstract}
Semiempirical molecular orbital calculations have been performed for the first step in the alkaline hydrolysis of the neutral benzoylester of cocaine. Successes, failures, and limitations of these calculations are reviewed. A PM3 calculated transition state structure is compared with the PM3 calculated structure for the hapten used to induce catalytic antibodies for the hydrolysis of cocaine. Implications of these calculations for the computeraided design of transition state analogs for the induction of catalytic antibodies are discussed.
\end{abstract}

Keywords: cocaine, electrostatic potential, ester hydrolysis, molecular orbital calculations, transition state, catalytic antibodies, transition state analogs

\section{Introduction}

The rational production of antibodies able to catalyze a particular reaction was first described by Lerner and Benkovic, $[1,2]$ and by Schultz, [3] in 1986. Their insight stemmed from a recognition of a structural similarity between an enzyme transforming a substrate and an antibody binding to a molecule that resembles the transition state structure of the substrate. [4] Such an antibody was found to stabilize the transition state over the ground state structure and lower the energy of activation for the indicated reaction. To elicit a catalytic antibody, the molecule that mimics the transition state, the so-called transition state analog (TSA), must be rendered immunogenic by being tethered as a hapten to a larger "carrier" molecule such as bovine serum albumin. Only a subpopulation of anti-analog antibodies will be catalytic and, in order to analyze the individual antibodies of a polyclonal immune response, monoclonal antibodies must be prepared and purified. The art of generating catalytic antibodies lies in the design of the hapten which must be chemically stable yet mimic the structural and electronic properties of the transition state of the indicated reaction.

One of us (DWL) has generated a catalytic antibody for the hydrolysis of the benzoylester of cocaine [5] and recently reported an improved catalyst that enhances the rate by 23,000-fold. [6] The hapten used for the generation of this catalytic antibody is based on the phosphonate monester structure, a structure used extensively for eliciting artificial esterases. [1, 3, 7, 8] In general, researchers designing cata- 
lytic antibodies for ester hydrolysis have focused on the general structure of the tetrahedral anionic intermediate as a model for the phosphonate hapten. $[1,3,7,8]$ The formation of the anionic intermediate is the first step in the alkaline hydrolysis of esters [9, 10 see Fig 1], and this is the ratelimiting step in solution. [11-13]

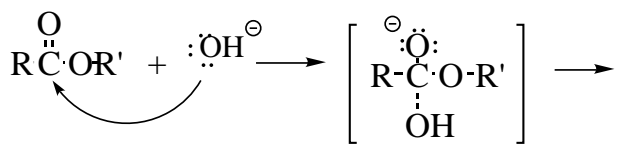

Reactants Transition State

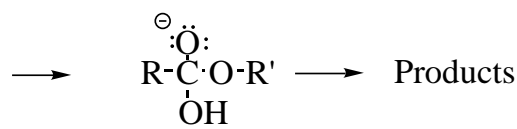

Intermediate

Figure 1. The first step in the alkaline hydrolysis of esters.

In our previous work extensive semiempirical and $a b$ initio computations were performed to model the first step in the alkaline hydrolysis of the model ester systems, methyl acetate and methyl benzoate. $[14,15]$ By comparison with high level $a b$ initio calculations at the HF and MP2 levels, it was found that the PM3 method showed promise for modeling transition state structures for ester hydrolysis. Other group's efforts to use quantum mechanics to calculate transition states in order to aid in understanding catalytic antibody work include studies on the chorismate-prephenate rearrangement, [16] the Diels-Alder reaction, [17] the aldol reaction, [18] and the ene reaction between malieimide and 1-butene. [19]

In this article the results of semiempirical calculations are presented for the alkaline hydrolysis of the neutral cocaine benzoylester, for the phosphonate hapten that has been used to generate a catalytic antibody that catalyzes cocaine hydrolysis, [5, 6] and for a newly designed thiophosphonate analog.

\section{Method}

The AM1, [20] MNDO, [21] and PM3 [22] semiempirical molecular orbital methods, implemented within the SPARTAN software package, [23] have been used to search for the transition state for the alkaline hydrolysis of cocaine. Starting structures were based on previous work, $[14,15]$ and careful searches for the transition state structure were made with each method. Because of the difficulties in obtaining a transition state structure, the methodology used in our calcu- lations will be described in the Discussion section of this paper. In addition the energetics of the critical first step were evaluated using the SM3 method [24] and compared with the methyl acetate and methyl benzoate systems.

All geometry-optimized structures were characterized as stationary points, and as minima or maxima, at all levels of theory in this study. The display of normal modes of vibration within SPARTAN allowed verification that the calculated transition structure connected reactants with the anionic tetrahedral intermediate. Molecular electrostatic potentials (MEPs) were calculated using the NDDO approach within SPARTAN. All calculations were carried out on Silicon Graphics Indy and Indigo ${ }^{2}$ workstations at Lake Forest College.

\section{Results}

The critical first step in the alkaline hydrolysis of esters is presented in Figure 1. Figure 2 displays the PM3 calculated transition state structure that connects the reactants and tetrahedral intermediate (see Figure 1). Figure 3 displays a PM3 calculated phosphonate analog that was the basis for the hapten used by Landry and co-workers to induce catalytic antibodies. [5, 6] The MEPs, superimposed on the electron density, for the transition state structure and the phosphonate analog are shown in Figure 4. The molecular framework of each structure is visible beneath the MEPs. Red indicates regions with the most negative electrostatic potential while blue indicates regions with the least negative electrostatic potential. Figure 5 shows the neutral cocaine ester and its highest occupied molecular orbital (HOMO). Cocaine and its lowest occupied molecular orbital (LUMO) are displayed in Figure 6. The thiophosphonate transition state analog, and the MEP superimposed on the electron density, are displayed in Figure 7. Table 1 contains the energetics for the reaction

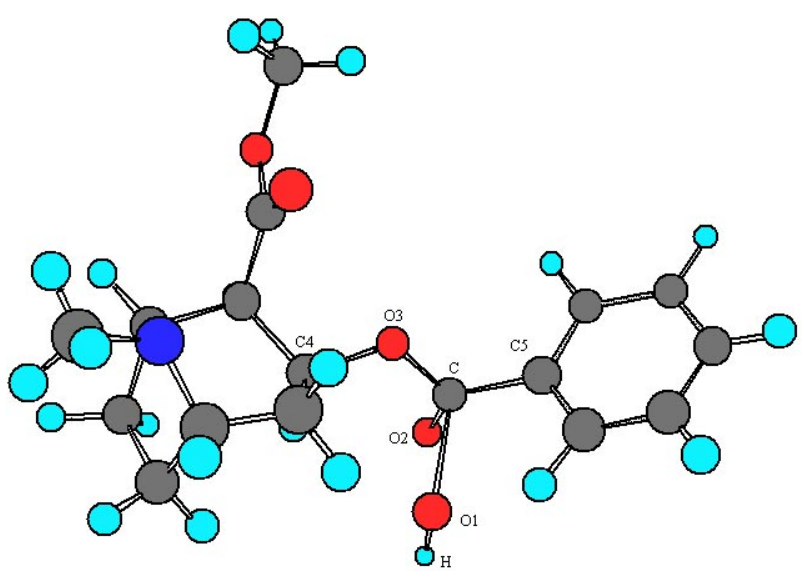

Figure 2. The PM3 calculated transition state structure for the first step in the alkaline hydrolysis of the neutral cocaine benzoylester. 
displayed in Figure 1, for cocaine and the previously determined ester systems. [14, 15] Geometric parameters for the transition state structure and the phosphonate hapten are listed in Table 2. Close contacts are shown for the carbon that is being attacked by the hydroxyl anion in the transition state structure and for the phosphate in the hapten molecule.

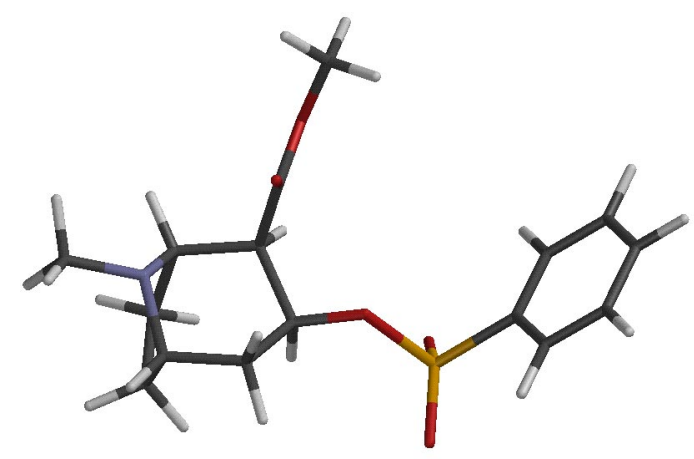

Figure 3. The PM3 calculated phosphonate analog, PA2, the hapten used to induce catalytic antibodies (ref 5,6).

\section{Discussion}

\section{Transition State Structure (TS)}

Previous computational work on the hydrolysis of the esters methyl acetate and methyl benzoate guided our search for the PM3 transition state structure (TS) for the hydrolysis of cocaine. [14, 15] For methyl acetate, the PM3 method located two transition states, one with a linear geometry and one with a bent geometry. The linear and bent geometries were characterized by the $\mathrm{C}$ (acetate methyl carbon)- $\mathrm{C}$ (acetate ester carbon)-O - $\mathrm{C}$ (methyl carbon) torsion angle of the transition state structure. Hartree-Fock (HF) ab initio calculations at the 3-21G/HF and 3-21+G/HF levels, starting from the two PM3 transition state structures, converged on a linear TS. Compared with the $3-21+\mathrm{G} / \mathrm{HF}$ structure, the linear PM3 TS was superior to the 3-21G/HF TS. [14] For methyl benzoate, the PM3 method found one linear TS. [15] AM1 calculations were not successful for obtaining a reasonable TS for either model system.

PM3 calculations were performed on the neutral cocaine ester, the hydroxide anion, and the intermediate formed after the first step in the alkaline hydrolysis of the cocaine ester. The anionic tetrahedral intermediate had a heat of formation of $-216 \mathrm{kcal} / \mathrm{mol}$, a $\mathrm{C}$ (acetate ester carbon)-O (hydroxyl oxygen) reaction bond distance of $1.414 \AA$, and a C-C-O-C torsion angle of $133.9^{\circ}$. The procedure that was successful for finding a PM3 TS was obtained through five steps. In step one the intermediate was used for a constrained transition state optimization, where the $\mathrm{C}-\mathrm{O}$ reaction bond dis- tance was subject to the constraint of being fixed to $2.30 \AA$. In addition the converge option was selected to aid SCF convergence. The output file for this calculation revealed that after 18 cycles of constrained transition state optimization, the gradient dropped to 0.007 a.u. and the heat of formation climbed to $-165.4 \mathrm{kcal} / \mathrm{mol}$. Further cycles increased the gradient. In the second step the same procedure was repeated, but reduced to 18 optimization cycles, to obtain the lowest gradient/highest heat of formation structure. In the third step a frequency calculation was performed on the 18 cycle output structure, by numerical differentiation of analytical gradients using central differences. One imaginary frequency was found $\left(-360 \mathrm{~cm}^{-1}\right)$ and examination of this normal mode revealed that this vibration connected the cocaine ester and hydroxyl anion reactants with the tetrahedral anionic intermediate. In step four the Hessian matrix, wavefunction, and structure from the previous step were used for a transition state optimization, again with the converge option but with the $\mathrm{C}-\mathrm{O}$ constraint removed. The output structure from this final optimization converged, had a heat of formation of $183.2 \mathrm{kcal} / \mathrm{mol}$, and was used for a frequency calculation in step five. One imaginary frequency was obtained $(-273 \mathrm{~cm}-$ 1) and examination of this mode revealed that this was the correct TS for this reaction. Furthermore, geometry optimizations performed from the TS, purturbed slightly along the transition state mode (first contracted, and second expanded), led first to the stable cocaine intermediate and second to the initial ester and hydroxyl anion reactants. The final TS is displayed in Figure 2.

This structure was used as the input structure for AM1 and MNDO transition state calculations. In addition the same strategy outlined above was used starting from the AM1 and MNDO minimized tetrahedral intermediates. Also, the linear synchronous transition (LST) method was used within SPARTAN to generate many different starting models for AM1 and MNDO transition state optimization, with the same results as described previously. [14] Efforts with these other semiempirical methods were not successful.

\section{AM1 vs PM3}

Failure of the MNDO method to find a TS is not surprising, given the known overestimation of the repulsive core-core potentials. [21,25] In order to reduce the repulsive forces between atoms separated by van der Waals distances, Dewar and co-workers modified the core repulsion function (CRF) by adding two to four parameterized Gaussian terms for each atom. This did indeed reduce the repulsive forces and has been found to be more useful for finding transition state structures than MNDO. In the PM3 Hamiltonian, two Gaussians were added per element in the CRF, and all of the Gaussian parameters were optimized within the PM3 framework. [22] The central difference between AM1 and PM3 is the parameterization. Why then is it possible for PM3 calculations to find transition states for the first step in the alkaline 
hydrolysis of cocaine ester, methyl acetate, [14] and methyl benzoate, [15] while AM1 calculations fail for these same systems? The answer does seem to reside in the difference between the parameterizations. It has been demonstrated previously that the PM3 parameterization is the reason why PM3 is better than AM1 for modeling intermolecular hydrogen bonding between neutral molecules, a consequence of the reduction of the overall repulsive forces between atoms by the parameterization of the attractive and repulsive Gaussian terms added to the CRF. [25] While the MNDO Hamiltonian drastically overestimates repulsions between atoms separated by van der Waals distances, and the AM1 Hamiltonian slightly overestimates repulsions between non-bonded atoms, the PM3 Hamiltonian underestimates repulsions between non-bonded atoms. This can be seen by the slight underestimation of PM3 determined hydrogen bond lengths [25] and by the formation of close atom-atom contacts such as in the methane dimer, where the H,H separation distance is $1.72 \AA$. [26]

There are other examples where the PM3 method allows non-bonded atom-atom distances to be too small. [13, 14, 26] The methyl benzoate TS has a hydrogen that is covalently attached to a carbon on the benzoyl ring that is $1.65 \AA$ from the incoming hydroxyl oxygen. Likewise, for the cocaine transition state structure shown in Figure 2, the same nearest hydrogen from the benzoyl ring is separated by $1.709 \AA$ from the incoming hydroxyl oxygen. In addition the closest hydrogen on the tropane ring is $1.736 \AA$ away from the incoming hydroxyl oxygen. These close contacts stabilize the PM3 $\mathrm{TS}$, but are repulsive interactions within the MNDO and AM1 Hamiltonians. Figure 2 shows a view of the cocaine ester transition state where the closeness of the two hydrogen atoms to the hydroxyl anion is apparent. Thus the PM3 method is successful for obtaining transition state structures for alkaline ester hydrolysis because of the stabilizing influence of non-bonded atoms, a consequence of the parameterization.

It is of interest to compare small basis set ab initio HF calculations on transition state structures of the methyl acetate system. We were not able to obtain a TS for methyl acetate using STO-3G/HF theory. Therefore the use of STO-
Table 1. Energetics for the first step in the alkaline hydrolysis of neutral cocaine, methyl acetate (ref. 14), and methyl benzoate (ref. 15) esters (see Figure 1). Gas phase results were obtained from the PM3 geometry-optimized structures. Solution results were obtained by SM3 single point calculations on the gas phase geometries. All energies in kcal/mol.

\begin{tabular}{lllll}
\hline System & $\begin{array}{c}\mathbf{E}_{\mathbf{A}} \\
\text { (gas phase) }\end{array}$ & $\begin{array}{c}\mathbf{E}_{\mathbf{A}} \\
\text { (solution) }\end{array}$ & $\begin{array}{c}\Delta \mathbf{H}_{\mathbf{r x n}} \\
\text { (gas phase) }\end{array}$ & $\begin{array}{c}\Delta \mathbf{H}_{\mathbf{r x n}} \\
\text { (solution) }\end{array}$ \\
\hline $\begin{array}{l}\text { methyl } \\
\text { acetate }\end{array}$ & -15.6 & 23.4 & -50.6 & 2.6 \\
$\begin{array}{l}\text { methyl } \\
\text { benzoate }\end{array}$ & -22.3 & 21.8 & -55.0 & 0.6 \\
$\begin{array}{l}\text { cocaine } \\
\text { ester }\end{array}$ & -28.5 & 24.6 & -61.4 & 0.4 \\
\hline
\end{tabular}

3G/HF methods for larger ester TS structures will probably not be useful. The 3-21G/HF transition state structure has two hydrogen-oxygen close contacts. The methyl hydrogen closest to the hydroxyl oxygen is separated by a distance of $1.827 \AA$ while the closest acetate methyl hydrogen is separated by a distance of $2.009 \AA$. By comparison these same separation distances are 2.301 and $2.459 \AA$ from $3-21+\mathrm{G} / \mathrm{HF}$ results and 2.44 and $1.83 \AA$ from PM3 calculated structures. Thus improving the PM3 cocaine transition state structure will require ab initio Hartree-Fock geometry optimizations that include diffuse functions. For the cocaine system, using Gaussian 94 [28] on an Indigo2 R4400 processor, one SCF calculation on the cocaine anion intermediate at the $3-21 \mathrm{G} /$ HF level requires 41.5 CPU minutes, and one SCF calculation at the $3-21+\mathrm{G} / \mathrm{HF}$ level requires $11.3 \mathrm{CPU}$ hours. The fact that the PM3 structures are as good or better than the $3-21$ G/HF structures gives confidence in the use of the PM3
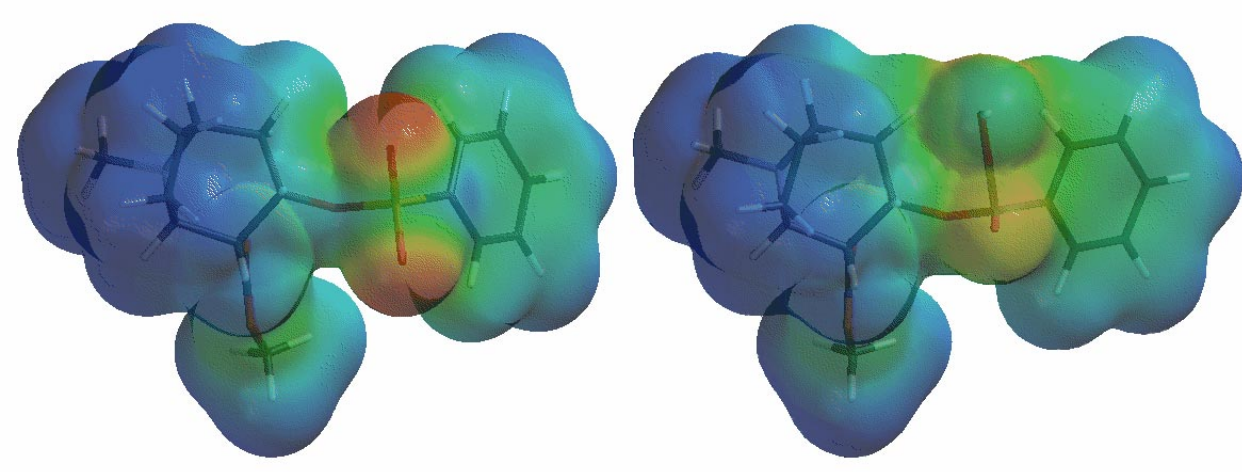

Figure 4. The PM3 calculated molecular electrostatic potential $(M E P)$, mapped onto the transparent electron density surface, and displayed for the transition state structure and the phosphonate analog. The molecular framework of each molecule is visible through the MEPs. The phosphonate analog, PA2, is displayed on the right side of the figure. Red indicates areas with the most negative electrostatic potential. 
TS structure for modeling alkaline hydrolysis of the neutral cocaine molecule.

Energetic comparison of cocaine and smaller ester systems

Table 1 contains the PM3 estimates for the activation energies and enthalpies of reaction for the first step in the alkaline hydrolysis of the three ester systems. Single point calculations were made (SM3//PM3) as our previous work revealed that SM3 geometry optimization did not result in substantial differences in these estimates. $[14,15]$ The observed activation energies for alkaline hydrolysis of esters in solution range from 12 to $15 \mathrm{kcal} / \mathrm{mol}$. [29, 30] The large cocaine benzoylester has a negative activation energy for gas phase hydrolysis, which changes to a positive barrier in solution, a direct result of the requirement to desolvate the hydroxide anion in solution. The dramatic lowering of the energy of the hydroxyl anion in solution also changes $\Delta \mathrm{H}_{\mathrm{rxn}}$ for the first step in alkaline hydrolysis, from a large exothermic reaction in the gas phase to approximately thermal-neutral in solution. The PM3 and SM3 values in Table 1 for cocaine parallel those for methyl acetate and methyl benzoate. These estimates reveal that the benzoyl ester of cocaine is not a difficult ester to catalyze, and it should be possible to achieve large rates of catalysis if a catalytic antibody can be made from an appropriate TSA.

Comparison between the PM3 transition state structure and the phosphonate analog hapten

Table 2 shows the structural parameters for the transition state structure (TS) and two phosphonate analogs. The first ana$\log$, PA1, is the global minimum on the PM3 potential energy surface. The second analog, PA2 (Figure 3), was obtained by constraining the C4-O3-P-C5 torsion angle to $155.3^{\circ}$, the same value as the transition structure. This structure is just $0.4 \mathrm{kcal} / \mathrm{mol}$ higher in energy than PA1. Geometry optimization of PA2, after removing the torsion angle constraint, yields the PA1 minimum. The two phosphonate analogs have similar bond distances and bond angles. The RMS deviation between PA1 and PA2 is $0.530 \AA$. The RMS deviation between TS and PA1 is $1.127 \AA$ and between TS and PA2 is $1.097 \AA$. The conformational freedom of the phenyl ring results in the production of many antibodies. Antibodies generated by the phosphonate TSA were originally screened to find two that had significant effects on hydrolysis. [5] As PA2 is the PM3 structure that is most faithful to the PM3 transition state structure, the MEPs of these two structures have been compared in Figure 4.

The TS MEP has its maximum negative potential around the hydroxyl oxygen $(-189.8 \mathrm{kcal} / \mathrm{mol}$, reddest region), a negative potential of approximately $-151 \mathrm{kcal} / \mathrm{mol}$ around $\mathrm{O} 2$, and its least negative region of potential about the central carbon region on the hydroxyl hydrogen $(-89 \mathrm{kcal} / \mathrm{mol})$.
Table 2. Comparison of the PM3 geometries of a transition state structure for the first step in the alkaline hydrolysis of the neutral cocaine benzoyl ester and the phosphate analog used to induce catalytic antibodies for cocaine hydrolysis.

\begin{tabular}{lccr}
\hline PARAMETER $[a]$ & \multicolumn{2}{c}{ STRUCTURE $[b]$} \\
& TS & PA1 & PA2 \\
\hline
\end{tabular}

Bond Distance $(\AA)$

$\begin{array}{lrrr}\text { O3-C4 } & 1.426 & 1.379 & 1.378 \\ \text { X-O3 } & 1.387 & 1.792 & 1.792 \\ \text { X-C5 } & 1.502 & 1.908 & 1.908 \\ \text { X-O2 } & 1.222 & 1.501 & 1.497 \\ \text { X-O1 } & 2.313 & 1.498 & 1.501 \\ \text { O1-H } & 0.939 & \text { NA } & \text { NA }\end{array}$

Bond Angle (degrees)

$\begin{array}{lrrr}\text { C4-O3-X } & 117.3 & 118.0 & 119.6 \\ \text { O3-X-O2 } & 118.7 & 106.4 & 106.3 \\ \text { O3-X-O1 } & 95.5 & 106.7 & 107.2 \\ \text { X-O1-H } & 102.0 & \text { NA } & \text { NA } \\ \text { O2-X-O1 } & 96.6 & 123.6 & 123.7 \\ \text { O2-X-C5 } & 125.9 & 110.4 & 110.6 \\ \text { O1-X-C5 } & 96.3 & 110.4 & 110.2 \\ \text { O3-X-C5 } & 111.9 & 95.5 & 94.6\end{array}$

Torsion Angle (degrees)

\begin{tabular}{lrrr} 
C4-O3-X-C5 & -155.3 & 171.7 & $-155.3[c]$ \\
$\mathrm{H}-\mathrm{O} 1-\mathrm{X}-\mathrm{C} 5$ & -115.6 & $\mathrm{NA}$ & $\mathrm{NA}$ \\
$\mathrm{H}-\mathrm{O} 1-\mathrm{X}-\mathrm{O} 2$ & 11.7 & $\mathrm{NA}$ & $\mathrm{NA}$ \\
$\mathrm{C} 4-\mathrm{O} 3-\mathrm{X}-\mathrm{O} 2$ & 44.5 & 58.5 & 91.7 \\
$\mathrm{C} 4-\mathrm{O} 3-\mathrm{X}-\mathrm{O} 1$ & -56.2 & -75.1 & -42.4 \\
\hline
\end{tabular}

[a] $X=C$ for TS, $P$ for PA1, PA2

[b]TS is the transition state structure, PAI is the global minimum transition state analog structure, PA2 is the transition state analog with the C4-O3-X-C5 dihedral angle constrained to -155.3 degrees, NA not applicable. [c] constrained to -155.3 degrees.

The ester oxygen has a potential of approximately $-140 \mathrm{kcal} /$ mol while the bluest region in the TS MEP has a potential of $-30.28 \mathrm{kcal} / \mathrm{mol}$. PA2 has its most negative potential on the oxygen farthest from the ester $\mathrm{O} 3(-183.5 \mathrm{kcal} / \mathrm{mol})$, with the other oxygen having nearly the same potential $(-182 \mathrm{kcal} /$ $\mathrm{mol})$. The ester $\mathrm{O} 3$ atom is surrounded by electrostatic potential, with the most negative value in this region being approximately $-152 \mathrm{kcal} / \mathrm{mol}$. The bluest region in the MEP of 


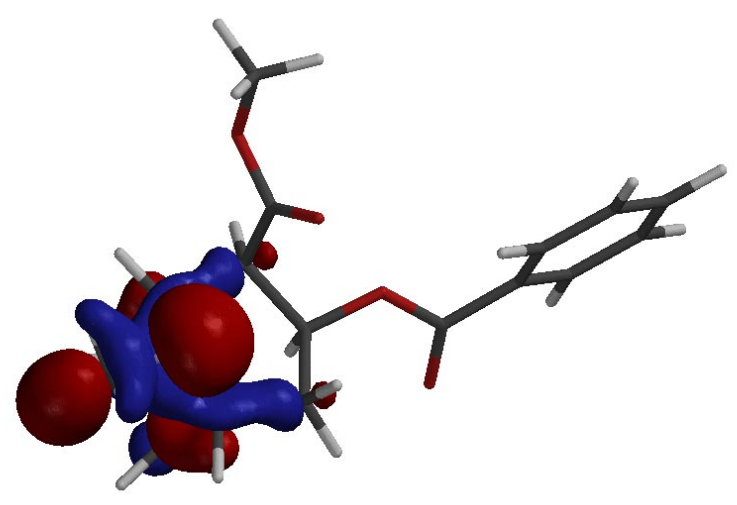

Figure 5. The PM3 calculated neutral cocaine ester and its highest occupied molecular orbital.

PA2 has a value of $-31.4 \mathrm{kcal} / \mathrm{mol}$. It is apparent that the phosphonate analog is more symmetric than the TS, and a clear strategy to improve the fidelity of the TSA to the TS is to design a TSA with asymmetric charge about the central atom. An example of this type of approach will be given in the next section.

\section{Implications for the design of better transition state analogs}

The TS for a gas phase reaction is usually different from the TS for the same reaction in solution and different from the same reaction catalyzed by an enzyme. Catalytic antibodies are induced by a TSA that resembles the TS, yet since a single TSA can elicit a multi-step catalytic activity it can be argued that the absolute fidelity of the TSA to the TS of an uncatalyzed reaction is not essential. For instance, Benkovic et al reported an interesting series of experiments where a phosphonamide TSA was used to induce a catalytic antibody (NPN43C9) that catalyzed both p-nitrophenylester hydroly-

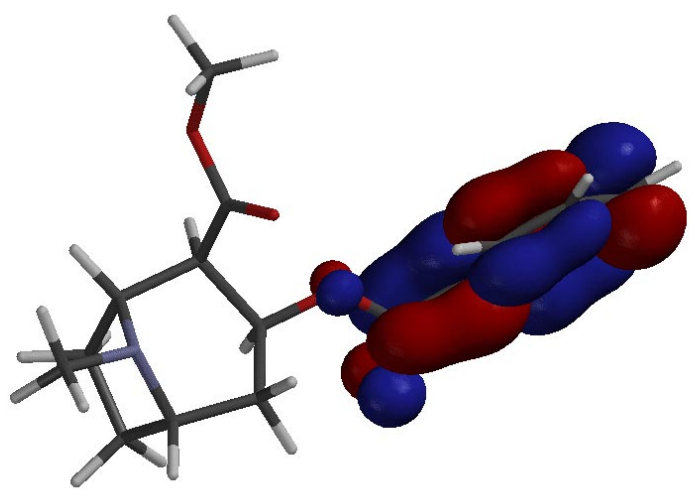

Figure 6. The PM3 calculated neutral cocaine ester and its lowest occupied molecular orbital. sis and p-nitroanilide amide cleavage. [31] Two points emerge from this work that are relevant to this discussion. First, the ester was catalyzed in a multi-step process, with a striking resemblance to the serine protease pathway. Both NPN43C9 and the serine proteases use a series of steps around an isoenergetic intermediate to achieve ester or amide cleavage. Second, the ultimate limit of ester hydrolysis was the rate of product dissociation from the catalytic antibody. This second point reveals that an important factor in hapten design should be to ensure that the TSA does not retain all the prominent structural features of the products, avoiding products that bind tightly to the catalytic antibody. Indeed, it has been shown that an analog that merely incorporates a relatively higher energy conformer is sufficient to produce efficient catalytic antibodies. [32]

Clearly there are many factors involved in the formation and the inner workings of catalytic antibodies that need to be understood. TheTSA is in an aqueous environment, and water must be displaced from the TSA for antibody induction. Thus the gas phase TSA, and by extension the gas phase TS, appear to be reasonable starting points for the computational problem of TSA design. The use of MEPs as a design aspect for modeling the TSA from the TS has precedent. A recent study of a TSA that is an inhibitor of an enzyme reveals that MEPs provide a clear explanation for the inhibition in this system. [33] In this investigation, the inhibitor formycin 5'phosphate binds to the AMP nucleosidase enzyme more tightly than the AMP substrate. Comparison of the MEPs of the inhibitor and substrate show a clear change in the electrostatic potential, a change that is matched by the MEP for the AM1 calculated transition state. The transition state for AMP hydrolysis is characterized by new positive electrostatic

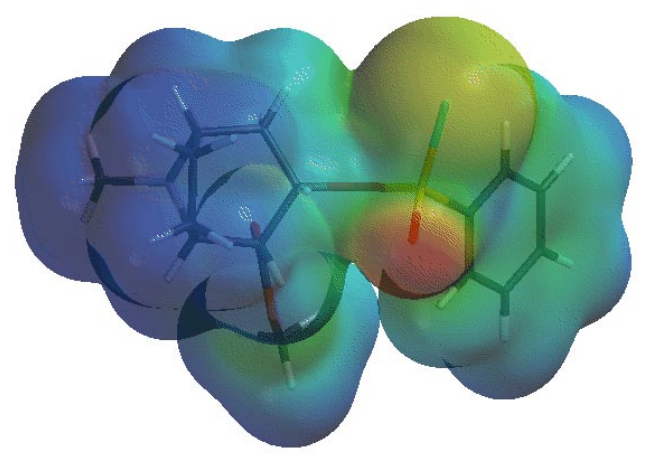

Figure 7. The PM3 calculated molecular electrostatic potential (MEP), mapped onto the transparent electron density surface, and displayed for the computationally designed thiophosphonate analog. The molecular framework is visible through the MEP. In this analog one of the oxygens on the traditional phosphonate TSA has been replaced through sulfur. 
potential in the adenine ring as a result of protonation by the enzyme. This is closely matched by the protonated pyrazole ring of formycin 5'-phosphate at position N7. The MEPs provide a clear explanation for the inhibition of AMP nucleosidase by the formycin 5'-phosphate TSA. This work illustrates that the use of MEPs of transition state structures to aid in the design of haptens is an idea that should be pursued.

The fact that a given TSA, such as the one used in the study by Benkovic et al, catalyzes a different reaction in a multi-step fashion highlights the complexity of antibody catalysis. One way that computational chemists can help is to use quantum mechanics to design rigorous TSAs, based on a property such as the MEP that is obtainable from the wave function, and then work with experimentalists to try and correlate the results with the quantum mechanical property. It remains an open question as to whether a rigorous TSA will generate a superior catalytic antibody, or whether methods such as bait and switch, [34, 35] or heterologous immunization, [36] are the optimal ways of generating antibody catalysts. An example of a designed TSA is the thiophosphonate analog displayed in Figure 7. In this analog one of the oxygens on the traditional phosphonate TSA has been replaced with sulfur. The effect of this substitution on the MEP is pronounced; the thiophosphonate MEP is asymmetric and appears to be a better match with the MEP of the TS structure. Differences remain, however, and work in our laboratories directed towards a quantitative comparison of the MEPs is underway. The thiophosphonate analog has been synthesized for use as a hapten for the induction of catalytic antibodies. Experimental work to characterize the molecular structure of this analog and its ability to induce catalytic antibodies is in progress. In addition we will pursue ab initio calculations to test the ability of PM3 to model this system and to compare the use of ab initio and semiempirical methods for modeling the crystal structures of the analogs.

The second point made by Benkovic et al was that the ultimate limit in ester hydrolysis in their multi-pathway system was the rate of product dissociation from the catalytic antibody. By comparing the MEPs of TSAs that do not structurally resemble the products with the MEPs of the TS, it may be possible to design haptens that are accurate TSAs yet do not contain the structural elements of the products. This in turn may lead to a catalytic antibody that has less product inhibition. This type of approach has been used by Miyashita et al to generate catalytic antibodies for the hydrolysis of a nonbioactive chloramphenicol monoester. [32] These ideas need to be tested through the interplay of computational predictions and experimental results using the computationally designed haptens. As an example of a way to use calculations to try and avoid slow product dissociation, consider the possibility of intramolecular catalysis. Figures 5 and 6 show that the PM3 HOMO is delocalized on the tropane nitrogen and most of the tropane ring, while the PM3 LUMO is delocalized over the benzoyl ester and the benzoyl ring for the neutral cocaine free base. Thus the electrons in the HOMO on the tropane $\mathrm{N}$ are poised for an attack on the LUMO, provided that the cocaine molecule can adopt a higher energy conformation that brings the tropane nitrogen and the benzoyl carbon into close proximity. Calculations can explore the conformational space of cocaine, find a TS for intramolecular catalysis, and model potential TSAs. As the TSAs for this system correspond to a high energy conformation of cocaine, it would be expected that there would be much less product inhibition of the cocaine molecule (Figs 5 \& 6) with the induced catalytic antibodies for intramolecular catalysis, similar to previous work. [32]

\section{Conclusions}

The neutral cocaine ester system is large and an anionic semiempirical transition state structure was only found for the PM3 method, a consequence of the parameterization of the method that underestimates non-bonded atom repulsion. Nevertheless, improvement on this anionic structure through $\mathrm{ab}$ initio calculations is going to require transition state optimization at the $3-21+\mathrm{G} / \mathrm{HF}$ level, as diffuse functions are essential for the proper description of ester hydrolysis [14, 15]. The PM3 calculated phosphonate transition state analog has an electrostatic potential comparable to the values for the PM3 calculated transition state structure, but the analog potential is more symmetric about the central $\mathrm{P}$, while the transition state potential is asymmetric about the central carbon atom. A newly designed thiophosphonate analog highlights the potential of the interplay between experiment and theory to enhance understanding of induction of catalytic antibodies by transition state analogs. The PM3 method has potential for the initial design of different analogs, and the experimental results obtained from using the computationally designed analogs should help unravel the most important quantum mechanical features in hapten design.

Acknowledgement. This work was supported by Lake Forest College, an NIH AREA grant, and an NSF ILI grant (GCS). This work was also supported by the Executive Office of the President of the United States, Office of National Drug Control Policy (DWL). We thank Gary Nalley for technical assistance. GCS acknowledges a sabbatical award from the Spanish Ministry of Education and Science and gratefully appreciates the hospitality of Professors Modesto Orozco and Javier Luque at the University of Barcelona while writing this manuscript.

Supplementary Materail Available: PDB files for all species are attached to this publication.

cocaine.pdb: PM3 geometry-optimized structure of neutral cocaine.

cocaine_intermediate.pdb: PM3 geometry-optimized structure for the anionic intermediate formed in the critical first step in the alkaline hydrolysis of cocaine. 
pa1.pdb: PM3 geometry-optimized structure of the phosphonate TSA used to induce antibodies for the hydrolysis of cocaine.

pa2.pdb: PM3 geometry-optimized structure for the phosphonate analog used to induce catalytic antibodies for the hydrolysis of cocaine, with the C-O-P-C torsion angle constrained to the same value as the PM3 calculated transition state, $-155.3^{\circ}$.

thio_phos_analog.pdb: PM3 geometry-optimized structure for the designed thiophosphonate analog. This analog has a MEP closer to the MEP for the PM3 TS structure than either of the phosphonate analogs, PA1 and PA2.

ts.pdb: PM3 geometry-optimized transition state structure for the critical first step in the alkaline hydrolysis of cocaine.

\section{References}

1. Tramontano,A.; Janda, K.D.; Lerner, R.A. Science 1986 234, 1566-1570.

2. Benkovic, S.J. Annu. Rev. Biochem. 1992 61, 29-54.

3. Pollack, S.J.; Jacobs, J.W.; Schultz, P.G. Science 1986 234, 1570-1573.

4. Lerner, R.A.; Benkovic, S.J.; Schultz, P.G. Science 1991 252, 659-667.

5. Landry, D.W.; Zhao, K.; Yang, G.X.-Q.; Glickman, M.; Georgiadis, T.M. Science 1993 259, 1899-1901.

6. Yang, G.; Chun, J.; Arakawa-Uramoto, H.; Gawinowicz, M.A.; Zhao, K.; Landry, D.W., J. Am. Chem. Soc. in press.

7. Tramontano, A.; Ammann, A.A.; Lerner, R.A. J. Am. Chem. Soc. 1988 110, 2282-2286.

8. Janda, K.D.; Benkovic, S.J.; Lerner, R.A. Science 1989 244, 437-440.

9. Bender, M.L.; Heck, H.d'A. J. Am. Chem. Soc. 1967 89, 1211-1220.

10. Shain, S.A.; Kirsch, J.F. J. Am. Chem. Soc. 1968 90, 5848-5854.

11. O'Leary, M.H.; Marlier, J.F. J. Am. Chem. Soc. 1979 101, 3300-3306.

12. Marlier, J.F. J. Am. Chem. Soc. 1993 115, 5953-5956.

13. Dewar, M.J.S.; Storch, D.M. J. Chem. Soc. Chem. Commun. 1995 94-96.

14. Sherer, E.C.; Turner, G.M.; Shields, G.C. Int. J. Quantum Chem., Quantum Biol. Symp. 1995 22, 83-93.

15. Turner, G.M.; Sherer, E.C.; Shields, G.C. Int. J. Quantum Chem., Quantum Biol. Symp. 1995 22, 103-112.

16. Wiest, O.; Houk, K.N. J. Org. Chem. 1994 59, 75827584.

17. Gouverneur, V.E.; Houk, K.N.; de Pascual-Teresa, B.; Beno, B.; Janda, K.D.; Lerner, R.A. Science 1993 262, 204-208.
18. Teraishi, K.; Saito, M.; Fujii, I.; Nakamura, H. Tetrahedron Lett. 1992 33, 7153-7156.

19. Yliniemela, A.; Konschin, H.; Neagu, C.; Pajunen, A.; Hase, T.; Brunow, G.; Teleman, O. J. Am. Chem. Soc. 1995 117, 5120-5126.

20. Dewar, M.J.S.; Thiel, W. J. Am. Chem. Soc. 1977 99, 4899-4907; 4907-4917.)

21. Dewar, M.J.S.; Zoebisch, E.G.; Healy, E.F.; Stewart, J.J.P. J. Am. Chem. Soc. 1985 107, 3902-3989.

22. Stewart, J.J.P. J. Comp. Chem. 1989 10, 209-221; 221264.

23. SPARTAN version 3.1, Wavefunction, Inc., 18401 Von Karman Ave., \#370, Irvine, CA 92715 USA.

24. Cramer, C.J.; Truhlar, D.G. J. Comp. Chem. 1992 13, 1089-1097.

25. Jurema, M.W.; Shields, G.C. J. Comp. Chem. 1993 14, 89-104.

26. Buß, V.; Messinger, J.; Heuser, N. QCPE Bull. 1991 11(1), 5.

27. Kirschner, K.N.; Shields, G.C. J. Mol. Struc. (THEOCHEM) 1996, 362, 297 - 304.

28. Gaussian 94 (revision B.1), M.J. Frisch, G.W. Trucks, H.B. Schlegel, P.M.W. Gill, B.J. Johson, M.A. Robb, J.R. Cheeseman, T.A. Keith, G.A. Petersson, J.A. Montgomery, K. Raghavachari, M.A. Al-Laham, V.G. Zakrzewski, J.V. Ortiz, J.B. Foresman, J. Cioslowski, B.B. Stefanov, A. Nanayakkara, M. Challacombe, C.Y. Peng, P.Y. Ayala, W. Chem, M.W. Wong, J.L. Andres, E.S. Replogle, R. Gomperts, R.L. Martin, D.J. Fox, J.S. Brinkley, D.J. Defrees, J. Baker, J.J.P. Stewart, M. HeadGordon, C. Gonzales, and J.A. Pople, Gaussian, Inc., Pittsburgh, PA 1995.

29. Amis, E.S.; Siegel, S. J. Am. Chem. Soc. 1950 72, 674677.

30. Rylander, P.N.; Tarbell, D.S. J. Am. Chem. Soc. 1950 72, 3021-3025.

31. Benkovic, S.J.; Adams, J.A.; Borders, Jr., C.L.; Janda, K.D.; Lerner, R.A. Science 1990 250, 1135-1139.

32. Miyashita, H.; Karaki, Y.; Kikuchi, M.; Fujii, I. Proc. Natl. Acad. Sci. USA 1993 90, 5337-5340.

33. Ehrlich, J.I.; Schramm, V.L. Biochem. 1994 33, 88908896.

34. Janda, K.D.; Weinhouse, M.I.; Schloeder, D.M.; Lerner, R.A.; Benkovic, S.J. J. Am. Chem. Soc. 1990 112, 12741275.

35. Janda, K.D.; Weinhouse, M.I.; Danon, T.; Pacelli, K.A.; Schloeder, D.M. J. Am. Chem. Soc. 1991 113, 54275434.

36. Suga, H.; Ersoy, O.; Tsumuraya, T.; Lee, J.; Sinskey, A.J.; Masamune, S. J. Am. Chem. Soc. 1994 116, 487494. 